\title{
LE GENRE NATIONAL COMME TRANSFERT CULTUREL
}

\author{
SCHLÄPFER, Anne-Frédérique ${ }^{1}$
}

\begin{abstract}
RÉSUMÉ : Cet article propose de considérer les relations entre la littérature francophone et les littératures étrangères en s'appuyant sur la notion de genre national. Bien que la théorie et l'histoire littéraires aient recouvert le concept de genre national par d'autres types de catégorisation, l'article cherche à montrer qu'il n'en a pas moins structuré les manières de penser la circulation mondiale de la littérature tout au long de la première partie de $\mathrm{XX}^{\mathrm{e}}$ siècle et qu'il constitue, dès lors, un exemple particulier de transfert culturel. En partant de l'exemple du « roman anglais », façonné dans le monde francophone entre le début des années 1920 et la fin des années 1940 à partir d'une vague de traductions, il s'agit de restituer comment la réception francophone a construit ce genre national, quels acteurs y ont participé et quels usages ils ont en proposé.
\end{abstract}

MOTS-CLES : Transfert culturel ; traduction ; genre national ; roman anglais ; littérature mondiale ; histoire littéraire.

\section{THE NATIONAL GENRE AS CULTURAL TRANSFER}

\begin{abstract}
This article considers the relations between francophone literature and foreign literatures, in regard with the notion of national genre. Although literary theory and history have disregarded the concept of national genre for the benefit of other types of categorization, the article seeks to show that it has nonetheless structured ways of thinking about the global circulation of literature throughout the first part of the twentieth century, and that it therefore constitutes an important example of cultural transfer. Using the example of the "english novel", shaped in the French-speaking world between the early 1920a and the late 1940s out of a wave of translations, the article aims at reconstituting the way the French-speaking reception constructed this national genre; at identifying which actors participated in it, and what uses of it they proposed.
\end{abstract}

KEYWORDS: Cultural transfer; translation; national genre; English novel; world literature; literary history.

\footnotetext{
${ }^{1}$ Anne-Frédérique Schläpfer est chargée de cours au Département de littérature française de l'Université de Genève, et boursière post-doctorale du Fonds national suisse de la recherche scientifique. Annefrederique.schlaepfer@unige.ch
}

Jangada | ano 9, nr. 17, jan/jun, 2021 | ISSN 2317-4722 
L'histoire de la littérature a vu, ces dernières décennies, ses contours changer. Le tournant transnational des études littéraires, dont découle la prise en compte croissante de l'importance de la traduction dans les canons nationaux, a profondément modifié les manières de penser et d'écrire l'histoire littéraire, qui prend désormais des accents globaux plus prononcés. Pourtant, ces approches peinent parfois à rendre compte de l'historicité des pratiques réelles et des conceptions de l'idée de littérature, du fait d'une focalisation sur des importations restreintes ou limitées dans le temps, ou parce qu'elles appliquent, aux périodes qu'elles prétendent éclairer, des modèles forgés pour et par d'autres époques. Dès lors, l'étude des transferts culturels et, partant, celle des modalités de la circulation de la littérature et de ses conceptions variables rendant compte du temps long des échanges, d'une part, et de la dissémination géographique large, de l'autre, s'avèrent particulièrement utiles à la compréhension des manières passées de penser la littérature.

J'aimerais, dans ces pages, réfléchir à l'importance des interactions de la littérature de langue française avec les productions mondiales, en revenant sur une catégorisation qui, bien qu'aujourd'hui largement oubliée, n'en a pas moins structuré la schématisation de la circulation de la littérature et, plus spécifiquement, celle de la traduction, au $\mathrm{XX}^{\mathrm{e}}$ siècle. Les «genres nationaux » ont en effet nourri la conceptualisation des importations de littératures étrangères jusqu'aux années 1950, et parfois au-delà, et esquissent une idée particulière de l'idée de littérature mondiale. Dans le cadre de cet article, je m'intéresserai plus précisément à la manière dont la littérature francophone a intégré les apports de la littérature étrangère, en m'appuyant sur le cas du « roman anglais $»^{2}$. Il s'agira de replacer ce $«$ moment anglais $»$ de la circulation du roman parmi la succession de vagues de traductions qui a enrichi le monde littéraire francophone depuis la fin du XIX ${ }^{\mathrm{e}}$ siècle. De fait, le «roman russe », le «théâtre scandinave » au XIX ${ }^{\mathrm{e}}$ siècle, mais aussi la «poésie belge », « les romans » anglais, américain, ou sudaméricain au $\mathrm{XX}^{\mathrm{e}}$ siècle, puis le «polar »scandinave au $\mathrm{XXI}^{\mathrm{e}}$ siècle - pour ne citer que ces exemples - ont tour à tour suscité l'intérêt des traducteurs, des éditeurs, des journalistes, des commentateurs comme des lecteurs francophones, et ont profondément modifié les pratiques des écrivains eux-mêmes.

Je chercherai à approfondir et à théoriser la notion de « genre national », en l'entendant comme un genre construit collectivement par la réception, au gré des œuvres traduites à une époque donnée, et à partir des discours qui les commentent. En cela, un genre national est une

\footnotetext{
${ }^{2}$ Je différencie, par l'usage des guillemets, le genre « roman anglais » de la production romanesque anglaise. Jangada | ano 9, nr. 17, jan/jun, 2021 | ISSN 2317-4722 


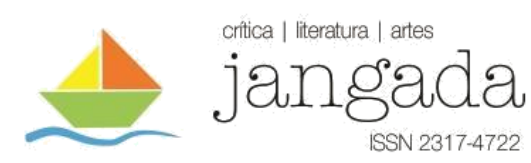

manière de donner sens et forme à la littérature étrangère, et fonctionne premièrement « à usage interne », puisqu'il répond aux préoccupations locales de chaque nation ou langue importatrice. En m'appuyant sur des travaux d'époque, et principalement sur les propositions d'Albert Thibaudet, fameux critique et professeur de littérature française durant l'entre-deux-guerres, je chercherai à montrer l'importance de la catégorisation de la littérature au prisme du genre, et la prégnance, - ou la résurgence - de l'idée de « genre national » dans les discours littéraires durant la première partie du $\mathrm{XX}^{\mathrm{e}}$ siècle. Cette période voit en effet se dessiner une nouvelle géopolitique littéraire, que l'on peut en partie corréler à la création de divers organisations et réseaux littéraires internationaux, tels que le Pen Club ou l'Institut international de coopération intellectuelle. S'ils se donnent pour mission la diffusion de la littérature à l'échelle mondiale, via la traduction, notamment, ils la pensent principalement en termes nationaux, en soulignant les spécificités esthétiques et culturelles de chaque nation littéraire, qui viennent enrichir un système mondial de genres nationaux.

Dans un second temps, je prendrai pour exemple le cas du « roman anglais », dont la construction, dans les années 1920, génère d'importantes discussions dans le monde culturel francophone, et ce jusqu'au seuil des années 1950. Ces débats nous renseignent non seulement sur la forte concurrence entre « roman français » et « roman anglais » dans les discours et sur le marché littéraires francophones ; ils révèlent aussi des conceptions plurielles, et parfois concurrentes, de la fonction de la littérature ; ils témoignent, finalement, des usages locaux du genre, que des auteurs français cherchent à s'approprier. Cette étude de cas m'amènera à préciser les méthodes susceptibles de rendre compte de la création d'un genre national, en croisant l'apport des études et de la théories littéraires, de l'histoire culturelle et de la traduction et de l'histoire globale.

\section{LE GENRE NATIONAL COMME TRANSFERT CULTUREL}

La question du « genre national » pose des difficultés aux spécialistes de la littérature actuels. Elles résident dans le fait de reconnaître une pertinence à une manière passée de construire des ensembles, qu'ils soient esthétiques ou théoriques. Il s'agira, ici, non seulement de renouer avec une catégorie, le genre, qui a suscité la méfiance du poststructuralisme - qui l'employait surtout pour montrer comment les textes la minent ou la débordent - mais aussi de l'associer à une dimension culturelle et nationale très étrangère à nos pratiques courantes. Et si cette suspicion 


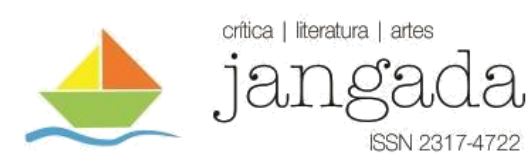

face aux genres littéraires est moins forte depuis le tournant des années 1990 qui a vu paraitre les importants travaux historiques de Nancy Armstrong (1987), de Margaret Cohen (1999) ou de Franco Moretti (2000) pour ne citer qu'eux ; si ce soupçon a été affaibli par les clarifications théoriques de Jean-Marie Schaeffer (1989) ou de celles de John Frow (2015), il demeure parfois compliqué de faire émerger et de réhabiliter un genre passé, d'autant plus lorsqu'il a été conçu selon des normes qui n'ont rien de commun avec l'idée que nous faisons aujourd'hui de la littérature. Il convient, dès lors, de restituer la façon dont la littérature était appréhendée dans les premières décennies du $\mathrm{XX}^{\mathrm{e}}$ siècle. Cristallisant la manière de penser la littérature propre aux années 1920-1930, le travail d'Albert Thibaudet me semble particulièrement représentatif - et même exemplaire - de cet attrait pour les genres dont il faut saisir les principes afin de mieux comprendre ce que pouvait signifier la notion de genre national à l'époque.

L'étude des interventions de Thibaudet parues dans la presse spécialisée et généraliste, et, depuis, reprises dans un important volume (2007), démontre qu'à sa réflexion sur le genre littéraire, le critique adjoint volontiers une contextualisation mondiale. Les articles qu'il consacre au « roman domestique » (THIBAUDET, 2007a) ou au « roman urbain » (THIBAUDET, 2007b), par exemple, illustrent sa façon de rapporter les genres littéraires à la production littéraire internationale. L'auteur explique en effet que les frictions et les « contradictions » entre les «formules» romanesques nationales sont constitutives de leur développement. A l'instar de nombre de ses contemporains, Thibaudet soutient l'idée selon laquelle « la vie littéraire française, comme la vie littéraire européenne ne vit que d'oppositions »(THIBAUDET, 2007a, p. 887). Commentant Le Justicier, un recueil de nouvelles de Paul Bourget, Thibaudet note ainsi que ces textes courts lui paraissent faire tenir ensemble «trois lignes anglaise, russe, française », dont le critique désire justement souligner « non leur accord, mais leur vie complémentaire et le dialogue de leurs oppositions » (THIBAUDET, 2007a, p. 887). L'évolution des formes dépendrait donc de la circulation de la littérature à une échelle supranationale davantage que de ruptures ou d'innovations élaborées dans un contexte proprement national.

Dans l'entreprise critique de Thibaudet, la description des genres ne dépend donc pas uniquement d'une nomenclature fondée sur des principes strictement esthétiques ou formels : elle s'inscrit dans une cartographie européenne, voire mondiale, du roman. Chaque nation apporte une conception particulière, un ton, des thèmes, des traits à ce système global. Le « goût des échanges »(THIBAUDET, 2007a, p. 890) modifie ainsi sensiblement la carte des imaginaires littéraires, qui ne s'arrête plus à la connaissance du seul « roman traditionnel Jangada | ano 9, nr. 17, jan/jun, 2021 | ISSN 2317-4722 
français »dont Paul Bourget serait le parfait représentant, mais implique une forme de comparatisme, si ce n'est de compétition entre différentes traditions romanesques. En ce sens, Thibaudet souligne les bienfaits du renouvellement apporté par d'autres nations à la tradition française du roman et, plus généralement, à toutes les formes culturelles qui, sans un tel voisinage, seraient susceptibles « de croupir dans le conformisme » (2007a, p. 890). Il ne fait aucun doute, pour le critique, que le roman se conçoit comme l'expression de traits culturels nationaux et que la concurrence entre les genres à l'échelle mondiale est tout à la fois esthétique et nationale.

Dans son effort de clarification des formes littéraires de l'époque, Thibaudet consacre un article entier au «roman anglais » après qu'a paru ce que le recenseur présente comme une « histoire du roman anglais » signée par Abel Chevalley (1921). C'est l'occasion pour Thibaudet de revenir sur le phénomène de l'internationalisation du roman qu'il ne cesse de discuter et dont il signale, ici, les fonctions majeures :

[L]e roman puise une de ses raisons d'être dans l'accouchement et l'éclaircissement des caractères nationaux, dans la mise au jour d'une Angleterre, d'une France, d'une Russie plus authentiques que les vraies ; il est le principal truchement qui fasse connaître les peuples les uns aux autres. Mais en même temps il tend à devenir un genre de plus en plus international. (THIBAUDET, 2007c, p. 589)

Le roman, «genre de plus en plus international », fait circuler entre les peuples, des « schématisations $»^{3}$ et des manières d'être issues des nations dont il est l'expression. La diffusion internationale du roman sert ainsi un idéal humaniste, en ce qu'elle contribue à familiariser les lecteurs avec l'altérité. Le roman, ainsi, se conçoit véritablement comme une forme commune, dans tous les sens du terme : d'abord parce qu'il supplante peu à peu les autres - la poésie et le théâtre - ensuite parce que ce genre est appropriable par des écrivains résidant dans des nations aussi diverses que l'Angleterre, la France ou la Russie. Sa plasticité le rend capable de transmettre à des publics variés des expériences et des manières de vivre différentes et jusque-là méconnues.

\footnotetext{
${ }^{3}$ « la vie humaine en groupe est faite de bien d'autres élans que celui de la famille, et ces élans, le roman a ses façon originales de sympathiser avec chacun d'eux, de le schématiser, de le revivre et de l'épanouir» écrit-il dans «Le roman urbain » (THIBAUDET, 2007b, p. 893).
} 
Si les travaux de Thibaudet témoignent d'un esprit de classification, et présentent une constellation riche de genres littéraires dont nous n'avons parfois plus mémoire (le « roman urbain », le « roman de la douleur », le « roman de l'énergie », le « roman-cycle »), ils nous renseignent également sur la portée de ce que j'ai choisi de nommer les « genres nationaux ». Leur théorisation progressive se prépare dès la fin du XIX ${ }^{\mathrm{e}}$ siècle et se généralise dans les années 1920, et emprunte volontiers le vocabulaire de la psychologie des peuples. Comme ses confrères, Thibaudet affirme, par exemple, qu' « [i]l y a des romans français et des romans anglais », tout en précisant ne pas croire en l'existence actuelle d' « un roman de l'Angleterre ou [d']un roman de la France » (THIBAUDET 2007b, p. 893). La différence qu'il impute à ces deux expressions, « roman anglais » et « roman de l'Angleterre », importe : elle tient dans l'acception du terme « roman ». Un « roman anglais » réunit des traits proprement anglais, qu'ils soient esthétiques ou culturels, alors qu'un « roman de l'Angleterre »-dont Thibaudet, faisant mine d'ignorer Scott, dit ne pas connaître d'exemple - doit se comprendre au sens de récit national.

Des interventions de Thibaudet, on aura compris que la circulation du roman accompagne et produit la progressive mondialisation culturelle, dont on peut situer les prémices vers 1870. Elle dessine une véritable « géopolitique des cultures nationales »(WILFERTPORTAL, 2002, p. 44) dans laquelle les vagues d'importation des littératures étrangères font à la fois office de modèle et de repoussoir pour chacune des nations littéraires. Ainsi, le « roman russe », la «poésie belge » ou le «théâtre scandinave », par exemple, ont joué un rôle déterminant dans la structuration des échanges culturels et dans la création des identités littéraires européennes au tournant des $\mathrm{XIX}^{\mathrm{e}}$ et $\mathrm{XX}^{\mathrm{e}}$ siècles. Ces genres nationaux, ou parfois régionaux, volontiers indexés à un double sentiment d'étrangeté - l'un relevant de la nouveauté formelle, l'autre d'une altérité culturelle, réelles, supposées, voire postulées - signalent tout autant l'émergence de nouvelles nations littéraires sur le marché que l'effort d'acclimatation concédé par les nations réceptrices en vue d'intégrer ou d'adapter ces productions à la culture locale. L'on peut envisager ces vagues d'importation comme la manifestation du désir de transférer les littératures présentant des avancées artistiques et culturelles et qui ont pris forme et se consolident dans une zone particulière de la carte du monde. En ce sens, l'innovation, en littérature, possède un centre fluctuant dont les vagues de traduction, aux fréquences plus ou moins rapides, diffusent les productions vers une constellation de nations réceptrices. Aussi, de vagues, c'est-à-dire, comme les qualifie Franco Moretti (2008), essors quantitativement mesurables d'une forme, ces importations deviennent vogues, à savoir, dans les termes de Jangada | ano 9, nr. 17, jan/jun, 2021 | ISSN 2317-4722 


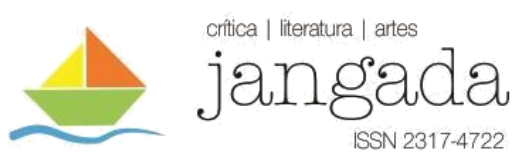

Christophe Charle (1994), discours, débats et usages locaux que l'on ne peut apprécier que qualitativement et à partir desquels se forgent les genres nationaux. Ainsi compris, ce phénomène d'importation massive indique d'une part la pression qui pèse sur les nations ou régions «périphériques », souvent peu dotées symboliquement, condamnées, comme la Scandinavie, la Russie ou la Belgique - à la « révolution symbolique » au sens que Pierre Bourdieu prête à ce terme (BOURDIEU, 2013, p. 377) pour entrer dans le système mondial et, de l'autre, la tension que cette nouvelle concurrence génère dans les nations dont la place est déjà établie, à l'instar de la France, qui diffuse largement sa production.

\section{« LE ROMAN ANGLAIS » : UN GENRE HISTORIQUE OUBLIE}

Comme le concept de genre national, la notion de « roman anglais » ne renvoie plus aujourd'hui qu'à un embarras théorique : elle oscille entre une catégorie historique inusitée, dont le flou générique et le caractère national suscitent d'emblée la suspicion, et un ensemble qui, bien qu'usuel, rassemble la production romanesque anglaise de manière si lâche qu'il en devient inopérant. Cet inconfort définitionnel et la difficulté à circonscrire l'épaisseur de la notion explique sans doute l'oubli dans lequel elle est tombée. Il faut dire combien le « roman anglais » échappe à notre manière actuelle, plus analytique, de catégoriser la littérature, à quelques exceptions près comme celle, déjà mentionnée, du «polar scandinave ». De fait, à son usage courant dans les années 1920-1950, et ce aussi bien auprès des critiques, des éditeurs, des journalistes que des écrivains et de leur public, s'est peu à peu substituée une série de notions concurrentes dont l'ampleur théorique et la fortune critique ont progressivement supplanté celle $\mathrm{du}$ « roman anglais ». Ainsi en va-t-il du modernisme, du modernisme tardif, du postmodernisme, du néo-romantisme, de l'impressionnisme littéraire, du roman poétique, du roman du courant de conscience, du roman subjectiviste, du roman polyphonique ou du monologue intérieur, entre autres. Si ces catégories délimitent des corpus et des périodes qui recoupent partiellement celles du « roman anglais », elles n'en demeurent pas moins principalement critiques - émanations de la théorie et de l'histoire littéraires de ces quarante dernières années - et ne tiennent pas, ou peu, compte des discours d'époque. Dès lors, elles opèrent un découpage temporel, esthétique et théorique tout différent de celui proposé par le genre historique $\mathrm{du}$ « roman anglais », tel qu'il est conçu dans le monde francophone.

Le caractère historique du « roman anglais » appelle plus largement l'adoption d'un point de vue anthropologique sur le phénomène des transferts (ESPAGNE, 2013, p. 305), et de Jangada | ano 9, nr. 17, jan/jun, 2021 | ISSN 2317-4722 


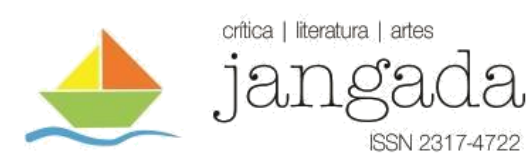

la circulation des formes littéraires, à même de le concevoir comme un fait culturel global. Pour cela, il convient de développer une méthode capable de rendre compte de la constitution du genre et de ses usages. Afin de procéder à ce que Margaret Cohen nomme «l'excavation » d'un genre (2009, p. 51), il importe de repérer, dans un premier temps, le spectre des débats qu'a suscités son apparition, puis d'en saisir les significations. Cela suppose de lire une constellation de productions d'époque signées de critiques, de préfaciers, de journalistes, de traducteurs et d'écrivains, mais aussi d'historiens et de penseurs dont les contributions ont nourri et façonné, pendant une trentaine d'années, l'imaginaire francophone du « roman anglais ». À cela doit s'ajouter une étude de ce qui se traduit à l'époque, c'est-à-dire ce sur quoi s'échafaude la théorisation du genre.

La situation de la traduction de l'époque mérite d'être étudiée, et fait apparaître plusieurs éléments. D'abord, le fait qu'entre 1920 et 1950, l'entreprise de traduction des œuvres qui viendront enrichir le « roman anglais », déjà largement amorcée depuis le tournant du siècle ce que démontrent les travaux de Blaise Wilfert-Portal - s'amplifie. L'historien montre que près de $47 \%$ des traductions parues en français entre 1926 et 1929 viennent de l'anglais (2003 : 93), ce qui constitue le taux d'intraduction, c'est-à-dire d'importation littéraire sous forme de traduction, le plus élevé pour une même langue dans le monde francophone. Le second élément porte sur la nature de ce qui est traduit : l'actualité littéraire ne discrimine pas fondamentalement les œuvres «classiques » ou « anciennes » des parutions récentes. Ainsi Walter Scott voisinet-il, entre autres, avec John Galsworthy, Virginia Woolf avec Jane Austen ou Conan Doyle. Ce dernier, d'ailleurs, connaît un nombre frappant de rééditions entre 1900 et $1925: 23$, alors que Dickens reparaît 51 fois, George Eliot 2 fois et Kipling 12, selon les chiffres établis par Wilfert (2003, p. 67). Paraissent donc des classiques, mais aussi des auteurs populaires, des auteurs de genre et des auteurs contemporains qui tous cohabitent, au même moment, sur les rayonnages des libraires francophones. On voit alors se croiser le temps long de ce que Bourdieu nomme la « production restreinte » et le temps court de la grande production. L'observation des effets de la traduction doit également rendre compte de la parution en français d'œuvres dont les auteurs rencontrent un succès moindre en Angleterre, alors qu'ils sont très appréciés par les lecteurs de langue française, à l'instar de Charles Morgan, très tôt traduits en français - et presque simultanément à leur parution en anglais, pour certaines œuvres.

En définitive, les œuvres qui constituent $\mathrm{du}$ « roman anglais » rendent les contours du genre plus complexe et protéiforme que ce que l'abord moderniste et canonique propre à 
l'histoire littéraire laisse entrevoir. Mais l'important réside surtout dans le fait que la constitution en genre s'opère à partir de cette multitude d'œuvres traduites dans lesquelles les commentateurs sont amenés à puiser pour sélectionner ce qui leur semble pertinent, et répond à des besoins locaux. Les œuvres passent donc à travers plusieurs filtres avant de venir grossir le réservoir du genre. Le comparatisme, l'humanisme, les pratiques de la traduction et les tensions du marché sont autant de conditions qui pèsent à des degrés variables sur la constitution du genre et sur sa compréhension.

Toutefois, l'importation de la littérature étrangère et, ici, celle des romans anglais ne dit rien de la prolifération concomitante des discours d'escorte. Or, à la différence des décennies précédentes, celles du tout début du $\mathrm{XX}^{\mathrm{e}}$ siècle, la traduction d'œuvres romanesques anglaises s'augmente massivement d'études savantes, d'articles de presse ou de recensions, mais aussi d'anthologies, de recueils d'analyses critiques et de panoramas souvent réédités. L'importation foisonnante, doublée d'un engouement critique et populaire engendrent donc, dès la fin des années 1910, une forme de théorisation qui transmue un ensemble perçu jusqu'alors comme relativement chaotique en un genre délimité, le « roman anglais ». Ce changement qualitatif et quantitatif n'est pas anodin et il faut y insister, afin de préciser une méthode susceptible de rendre compte de ce processus, allant du transfert, à sa théorisation et à ses justifications.

Appliquée au « roman anglais », l'étude quantitative ou la « lecture de loin » revendiquée par Moretti (2013), élude pour partie les raisons de l'importation et, surtout, les mécanismes de la construction de ce genre national qui s'originent ici dans une réception nationale ou linguistique spécifiques. Pour le dire dans d'autres termes, c'est la constitution en genre du flot de traductions qui forment le «roman anglais » que la mesure quantitative ne parvient pas à décrire. Ce que manque cette approche, ce qu'elle ne permet pas de rendre perceptible, c'est, finalement, la texture sociale et historique des formes que lui a forgée la réception. N'apparaît pas, dans ce type d'études, ce que l'on disait du genre, et qui l'énonçait, en somme : ce qui faisait débat. De plus, il est difficile de distinguer les phases de ces discours, des premières apparitions des traductions à leur appariement et identification en tant que genre. L'une des particularités des genres nationaux tient dans le fait que la nécessaire définition permettant leur reconnaissance dépend principalement de la nation, et/ou, de la langue importatrice. La catégorisation, ici, n'est pas prescriptive en premier lieu, en ce qu'elle ne modifie pas directement les sources anglaises, comme ont pu l'être les catégories génériques à l'époque classique qui, elles, réglaient strictement les usages. La catégorie ne pèse sur les manières d'écrire que dans un second temps, celui des réappropriations locales, et ce de façon Jangada | ano 9, nr. 17, jan/jun, 2021 | ISSN 2317-4722 


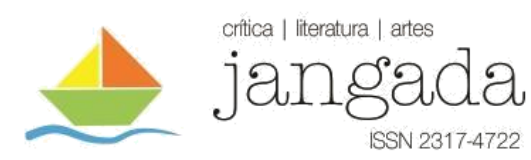

contrastive et dissociée - ne pas faire du « roman anglais » lorsqu'on se veut « écrivain français ", par exemple, ou, à l'inverse, revendiquer ce modèle pour échapper à d'autres étiquettes ou encore s'en approprier les traits pour les adapter à un nouvel espace.

Si la catégorisation n'est pas directement prescriptive, elle produit en revanche des effets plus directs, pragmatiques, sur les manières de lire les textes car, en instituant une croyance sur ce qu'est le « roman anglais », elle configure en partie la lecture des œuvres. Et c'est ce processus complexe qu'une approche s'attachant à restituer le point de vue des acteurs de l'époque dans les termes qui étaient les leurs permet d'exposer. En ce sens, le « roman anglais » $\mathrm{vu}$ « de près » devient un lieu commun où se rencontrent de nombreux acteurs majoritairement francophones - éditeurs, traducteurs, critiques, journalistes, écrivains et lecteurs - qui contribuent à forger conjointement cet objet culturel.

En m'inspirant des travaux de Marie-Ève Thérenty et de Dominique Kalifa (2016) qui proposent de comprendre comment la circulation transnationale des textes interagit avec la production locale, en ce qu'elle répond à une gamme d'enjeux sociohistoriques variés et spécifiques à chaque situation, j'aimerais ajouter à la focalisation macrologique de Moretti un point de vue micrologique. Si Thérenty et Kalifa insistent sur les usages locaux d'un genre transnational - en l'occurrence le mystère urbain au XIX ${ }^{\mathrm{e}}$ siècle - en montrant comment l'appropriation locale module la forme globale, ils s'attachent moins à décrire les constructions sociales, les débats et les prises de position plurielles que suscite une nébuleuse d'œuvres encore mal circonscrite, que l'implantation, dans un nouvel environnement, d'un genre déjà reconnu comme tel. Travaillant sur la dimension esthétique, les modifications stylistiques et les usages multiples de telle ou telle forme, ces chercheurs constatent qu'il existe pour un même genre autant de variantes et de significations qu'il y a de contextes. À cette épaisseur esthétique du problème, il me semble toutefois nécessaire d'en adjoindre une plus politique. C'est du côté des historiens de la culture, inspirés par la sociologie, que l'on peut trouver des travaux portant une attention particulière à la question des vogues des genres nationaux, c'est-à-dire à l'ensemble des débats qui structurent le transfert et la réception d'une forme étrangère. Si Christophe Charle, Michel Espagne et Blaise Wilfert-Portal, notamment, ont mis en évidence ce que les transferts culturels et l'importation massive de la traduction font au monde artistique et intellectuel qui les reçoit, ils n'adoptent pas la notion de «genre national ». Ce n'est donc pas tant sur les effets esthétiques que sur les frictions et les repositionnements politiques, sociaux et théoriques induits par les nouvelles formes qu'ils mettent l'accent. Ainsi décrivent-ils la grammaire des genres que chaque nation produit au fil de sa familiarisation avec ces formes Jangada | ano 9, nr. 17, jan/jun, 2021 | ISSN 2317-4722 
nationales étrangères. Les règles et les principes qui viennent à régir la compréhension des textes sont le fruit d'intenses débats qu'il importe de retracer.

Croiser les vagues et les vogues revient à coupler macro- et microhistoire, mais aussi histoire littéraire, histoire socio-politique et histoire globale. Cela nous amène donc à prendre en compte les formes et la pluralité des usages des transferts littéraires. Si les vagues sont l'expression de la circulation transnationale des formes sur la longue durée, les vogues sédimentent les usages esthétiques, intellectuels, théoriques, politiques et sociaux diversifiés qu'une forme peut générer localement. Ces variations d'échelles me permettent de penser la géopolitique de la littérature propre à l'époque. Au niveau macrologique d'observation, elle implique non seulement une concurrence transnationale entre les cultures littéraires, mais aussi un système transnational de genres nationaux qui s'institue progressivement. Aussi le genre national se comprend-il comme un «super-genre » qui fonctionne à un autre niveau et selon une autre logique que les sous-genres, par définition plus restreints, tels que le roman policier, le roman historique ou le roman sentimental. Moins prescriptif que prospectif, le genre national est filtré par la traduction, façonné par la lecture et les critiques ; en somme, construit collectivement selon les croyances et les valeurs d'une époque, d'une nation et d'une langue données. En ce sens, il est davantage révélateur de ce dont a besoin la nation réceptrice que de la production de la nation dont il émane. Un genre national a donc une double fonction. Celle repoussoir pour la culture qui l'accueille et le conceptualise, puisqu'il exige de sa part un questionnement global sur la littérature et ses usages, et contribue ainsi à en renouveler les définitions en vigueur. Celle de réservoir formel, en ce qu'il ouvre de nouvelles voies aux écrivains locaux.

\section{LA VOGUE DU « ROMAN ANGLAIS »}

Entre le début des années 1920 et le début des années 1950, on publie en volume des dizaines de textes critiques sur le « roman anglais », destinés, d'une part, à un public éclairé mais non spécialiste, auquel on propose des introductions générales à la littérature anglaise, et, de l'autre, aux premières générations d'universitaires anglicistes dont l'intérêt va davantage vers les monographies portant sur l'époque romantique ou sur des études savantes d'histoire littéraire. Cette pléthore de travaux coïncide également avec l'apparition de comptes rendus et de chroniques littéraires dans des journaux, souvent liés à des éditeurs. Ces journaux accordent 
une large place à l'actualité éditoriale française ou traduite ${ }^{4}$, promouvant ou décriant les œuvres selon leur affiliation, et créant ainsi un terreau favorable à la polémique ; cette masse de discours correspond également à la naissance de plusieurs revues proprement littéraires, comme $\mathrm{La}$ Revue de Genève (1920), Les Nouvelles littéraires (novembre 1922) ou Europe (février 1923), qui valorisent la littérature étrangère. À la pluralité des publics répond une diversification des acteurs de ce domaine critique, qui appartiennent à la diplomatie culturelle, à la grande bourgeoisie cosmopolite - dont les membres ont l'avantage de maîtriser les langues étrangères et de détenir des carnets d'adresses leur donnant accès aux réseaux transnationaux - ou, pour d'autres, aux mondes très poreux du journalisme, de l'édition ou de la traduction.

Parmi la multitude des parutions, signalons, par exemple, l'ouvrage d'Abel Chevalley, Le Roman anglais de notre temps (1921), et celui de Firmin Roz, Le Roman anglais contemporain (1921). Albert Thibaudet rassemble quelques-unes de ses critiques dans Étranger ou Études de littérature (1925) ; André Maurois donne un recueil de lectures, Études anglaises (1927), mais distille également ses réflexions dans la presse et dans plusieurs de ses journaux intimes, Relativisme (1930) et Fragments d'un journal (1931). Valery Larbaud fait paraître Ce vice impuni, la lecture. Domaine anglais (1925), qui sera édité à nouveau en 1936. Louis Gillet propose ses Esquisses anglaises (1930) et Edmond Jaloux publie Au pays du roman - entendez : l'Angleterre - en 1931. Les Approximations de Charles Du Bos sont publiées en plusieurs volumes tout au long des années 1930. L'écrivain genevois Guy de Pourtalès donne une série de conférences dans plusieurs villes suisses sur le « roman anglais » durant l'année 1937. Émile Saillens produit une Esquisse des littératures de langue anglaise. Grande-Bretagne, Irlande, Inde, Dominions, États-Unis (1938). Après-guerre, paraissent encore les Propos sur la littérature anglaise de Montgomery Belgion (1947). À ces livres, il faut ajouter les préfaces, les recensions et comptes rendus publiés dans la presse et les interventions publiques ponctuelles de ces hommes de lettres, tout à la fois écrivains, intellectuels, critiques et journalistes, parfois mêmes éditeurs et traducteurs. Pensons notamment au Suisse Jacques Mercanton, commentateur prolixe de Joyce, à René Lalou, préfacier, entre autres, de Woolf, D.H. Lawrence ou Morgan dans les années 1930, à André Rousseaux (1938) ou à Claire-Éliane Engel (1946 ; 1949). Ces divers travaux herméneutiques sont complétés par un abondant corpus

\footnotetext{
${ }^{4}$ En France citons : Candide (1924), Comøedia (1907-1914 puis 1917-1937), La Gazette de Paris (1928), Gringoire (1928), Marianne (1932), 1933 : Le Magazine d'aujourd'hui (1933), Vendredi (1935) mais aussi, en Suisse : La Bibliothèque universelle (dans sa nouvelle mouture de 1909 à 1922), La Revue de Genève (1920-1930), La semaine littéraire (1893-1927), La Revue Aujourd'hui (1930-1932).
} 


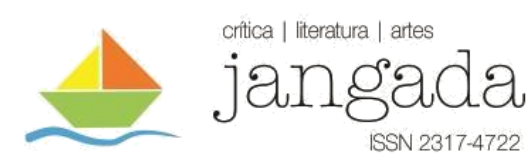

de biographies et de correspondances qui visent à approfondir la connaissance que les lecteurs francophones peuvent avoir des auteurs anglais ; des sœurs Brontë à Katherine Mansfield ou D. H. Lawrence, nombreux sont les ouvrages qui réunissent et éclairent la vie et l'œuvre de ces écrivains. Ces parutions s'inscrivent dans un mouvement amorcé par plusieurs grands éditeurs parisiens : Plon, Grasset ou Gallimard proposent en effet des collections spécialement dédiées au domaine biographique dans l'idée qu'elles fournissent des clés de conduite morale à un lectorat réputé en perte de repères.

Si le monde de l'édition connaît une vive effervescence autour du « roman anglais », l'université n'est pas en reste : on y présente de nombreux travaux académiques, monographiques, comme Le Roman psychologique de Virginia Woolf (1932), par Floris Delattre. D'autres portent sur des aspects plus généraux et historiques, comme la somme de Louis Cazamian et Émile Legouis, Histoire de la littérature anglaise (1924), le livre de Paul Dottin, La Littérature anglaise (1931), l'ouvrage de Madeleine Cazamian, Le Roman et les idées en Angleterre (1923), celui d'Aurélien Digeon, Histoire illustrée de la littérature anglaise (1947), ou encore le travail d'Irène Simon, Formes du roman anglais de Dickens à Joyce (1949). Cet afflux de travaux universitaires témoigne de la professionnalisation des anglicistes français qui, depuis 1905, se spécialisent dans la recherche en histoire littéraire et font souvent correspondre la production littéraire nationale anglaise avec l'idée qu'ils se font de l'âme de cette nation. Outre la publication de divers manuels destinés aux étudiants, la création de revues spécialisées, comme La Revue Études anglaises, fondée en 1937 à la suite de La Revue angloaméricaine lancée, elle, par des professeurs de la Sorbonne en 1923, signe la variété des entreprises éditoriales menées à l'époque pour promouvoir et institutionnaliser la littérature anglaise. La diffusion de ce savoir nouveau est également privilégiée par des séries de conférences publiques.

Bien que la prolifération de la notion de « roman anglais » se construise principalement dans le milieu francophone, elle s'alimente parfois d'essais d'écrivains anglais qui entrent, volontairement ou non, dans le débat. Ainsi les essais de Woolf, E. M. Forster ou John Middleton Murry font-ils l'objet de commentaires et de discussions animées, voire polémiques. Dans un article paru dans La Nouvelle Revue française, T. S. Eliot (1927) s'en prend directement au point de vue que défendait Chevalley quelques années plus tôt, dans Le Roman anglais de notre temps, témoignant par là de l'intérêt porté, de part et d'autre de la Manche, à la construction différenciée des imaginaires littéraires nationaux. La prédominance francophone n'empêche donc en rien le dialogue et la fabrication transnationale du genre - une Jangada | ano 9, nr. 17, jan/jun, 2021 | ISSN 2317-4722 330 | Pá g in a 
semblable démarche est opérée, par exemple, dans la sphère germanophone dans les mêmes années - bien que les définitions et les usages locaux priment toujours, dans le cas présent, sur leurs compréhensions anglaises.

La conceptualisation du genre du « roman anglais », je l'ai dit, relève principalement, dans le cas qui m'occupe, d'un phénomène de réception, c'est-à-dire, pour parler avec Gérard Genette, d'une attention critique plutôt que d'une intention d'auteur (1994, p. 245). La normalisation du genre - entendue à la fois comme codification et comme acclimatation - a d'abord servi la description des nouvelles techniques explorées par des auteurs comme Henry James, Huxley, Joyce ou Woolf, dont les œuvres, introduites dans le monde francophone dans les années 1920 et 1930, justifiaient et réclamaient de manière pressante une mise en perspective théorique et critique. Dans cette perspective, la classification aurait pour première ambition de rendre l'abondance et l'originalité intelligibles, c'est-à-dire, récupérables. Cette hypothèse forte a été développée par Michel Raimond dans un livre majeur (1966) qui met en lumière ce qu'il nomme la « crise du roman » que connaît la France dans les années 1890-1920 suite, notamment, à sa familiarisation avec la production romanesque anglaise et avec le cinéma. Pour Raimond, les techniques anglaises du monologue intérieur, de la multiplication des points de vue, du traitement de la temporalité ou de la caractérisation des personnages ont une profonde répercussion sur le champ littéraire français. Modèle ou repoussoir, la littérature anglaise engagerait in fine une reconfiguration de l'écriture romanesque dans le monde francophone, fondée sur une conception nouvelle de la réalité, du sujet individuel, de la mémoire et du temps.

Cette explication, qui s'attache avant tout à rendre compte d'évolutions esthétiques relatives à la composition romanesque, tout admirable et utile soit-elle, évacue toutefois une partie de ce qui faisait, aussi, l'intérêt du « roman anglais », en dehors de sa puissance de renouvellement technique. Comment comprendre cette reconstruction critique et quelles pertes occasionne-t-elle au juste ? De l'aveu même de l'historien de la littérature, les raisons et les enjeux des polémiques autour de la réception des œuvres anglaises lui apparaissent peu claires. Il convient de rappeler que Raimond écrit sa somme au milieu des années 1960, alors même que les techniques initiées par les romanciers anglais de la première moitié du siècle - et, en tête de ces dernières, le monologue - étaient déjà devenues les plus connues « du siècle » (MORETTI, 1996, p. 124) ou, à tout le moins, des « techniques parmi d'autres » (PHILIPPE, 1997, p. 424), largement reprises dans le roman mondial, et dont on pouvait même constater une résurgence dans les pratiques littéraires françaises des années 1960, notamment chez les Jangada | ano 9, nr. 17, jan/jun, 2021 | ISSN 2317-4722 


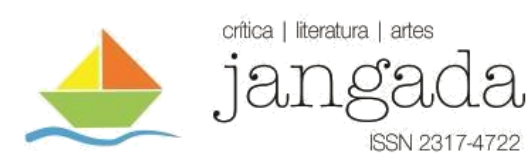

nouveaux romanciers. D’originales qu'elles étaient dans les années 1920-1930, ces techniques sont, dans le contexte littéraire des années 1960, habituelles. En se focalisant sur les techniques afin d'en montrer, d'une part, l'étrangeté pour un lectorat alors peu préparé et, d'autre part, la diffusion dans le roman francophone, Raimond entraîne, en réalité, le démembrement de la catégorie du « roman anglais », qui, pour lui, ne constitue aucunement un genre, mais bien davantage un réservoir de techniques dont on peut repérer l'agencement variable dans les textes. Par l'ampleur des phénomènes étudiés, l'analyse de Raimond éclaire magistralement les paramètres de la mutation esthétique qui se déploie alors. Mais, en dotant les acteurs de l'époque de termes et de catégories critiques dont ils ne disposaient pas, en remplaçant leurs « critères inadéquats » peu à même de rendre compte d' « exigences nouvelles encore confuses » (RAIMOND, p. 11-12) par le vocabulaire de la narratologie et du structuralisme des années 1960, bref, en rationalisant des pratiques diffuses et hésitantes, analysées une à une, il surdétermine la part des auteurs modernistes et de leurs techniques dans le vaste lot des traductions disponibles, et évacue, ce faisant, un aspect important de ce que signifiait le « roman anglais ».

Restituée à la «confusion » et à la nouveauté que produit le « roman anglais » à l'époque, la réaction de Thibaudet, qui qualifiait l'obsession de la critique française pour la composition de ce genre de «lieu commun » (THIBAUDET, 2007c, p. 596), s'éclaire en partie. Il met le doigt sur le fait que de nombreux débats opposaient au roman étranger, et anglais en particulier, un « défaut d'ordonnance »(BOURGET, 1922, p. 127). Celui-ci relèverait, selon eux, de l'artifice, tandis que le « roman français » serait, lui, tout de maîtrise et de mesure. Pour sortir des propos convenus, Thibaudet disqualifiait la question de la composition en en réservant la valeur au domaine théâtral. Il insistait, en revanche, dans l'ouverture de son article, sur l'immense succès planétaire rencontré par le « roman anglais »: «Non seulement les débouchés du livre anglais le déversent sur la large partie du globe dont l'anglais est la langue naturelle, mais son public français, germanique, slave, oriental et extrême-oriental, s'accroît sans cesse. » Le « roman anglais » n'est plus ici - du moins plus seulement - le vecteur privilégié du prestige littéraire ou de l'innovation, mais plutôt une formidable réussite économique et commerciale. Ainsi Thibaudet pointe-t-il, en creux, deux des raisons pour lesquelles l'analyse en termes de techniques échoue partiellement : l'importation ne touche pas uniquement les textes les plus novateurs, et le public s'attache à d'autres aspects du genre que sa seule qualité stylistique.

Cette deuxième explication se déploie donc en deux temps. J'aimerais défendre l'hypothèse selon laquelle il y a dans le « reste » laissé par le démembrement du « roman Jangada| ano 9, nr. 17, jan/jun, 2021 | ISSN 2317-4722 
anglais » opéré par Raimond une part fondamentale de ce qui faisait l'engouement pour le genre. Si les techniques nouvelles pouvaient, pour certains lecteurs, être l'une des raisons de l'intérêt suscité par le « roman anglais », elles ne pouvaient pas suffire à en expliquer le succès. Celles-ci ne se dévoilent en effet que relativement tardivement, au tournant des années 1930, alors même que la vague de traduction est déjà bien amorcée. En cela, l'exemple de l'Ulysse de Joyce est parlant. Publié à Paris en 1921 dans sa version originale, ce roman, que la critique contemporaine considère, non sans biais téléologique, comme le fleuron du modernisme et des explorations techniques et linguistiques - en somme, de «l'obscurité délibérée » (MORETTI, 1987, p. 27) - n'est traduit en français qu'en 1929. Les recherches dont atteste Ulysse n'ont donc été accessibles que relativement tardivement et ne peuvent avoir été décisives dans la propagation $\mathrm{du}$ 《 roman anglais » : elles représentaient certes une des facettes du genre, mais elles n'en étaient pas moins qu'un des éléments du « spectre de diversité » (MORETTI, 2008, p. 108) qu'il constitue. De nombreux commentateurs de l'époque, soucieux de rendre toute son épaisseur au genre, ont rappelé avec insistance la pluralité de ce qui se publiait alors : «il ne faut pas oublier que, parallèlement aux œuvres d'un genre nouveau, il n'a cessé de paraître des livres d'un mérite égal ou supérieur et conformes à l'esthétique traditionnelle. [...] Bennett, Wells, Shaw, Moore, n'ont transformé ni leur façon d'écrire, ni leurs idées, ni leur sens de proportion ; c'est la matière seule de leur observation qui s'est transformée » écrit Desmond MacCarthy pour la Revue de Paris (1932 : 130). En Suisse, Emmanuel Buenzod reprend cette même opposition entre manière moderne et manière traditionnelle pour rejeter la plupart des romans de Virginia Woolf au profit d'œuvres moins novatrices esthétiquement, comme celles de Stephen Hudson.

Ces techniques modernes ne font guère l'unanimité. Se dessine, parmi la critique généralement favorable au « roman anglais », une frange réfractaire à ces procédés, et qui y voit un moyen artificiel de rendre les textes « décousus»(VUILLIOMENET, 1932, p. 23). Mais la bataille entre modernes et classiques peut être désamorcée, ou déplacée, comme le fait Jaloux dans des pages consacrées à Gens de Dublin de Joyce, dans lesquelles le critique remarque que le succès récent d'un roman de Jean Paul, Quintus Fixlein, édité en allemand entre 1794 et 1795, mais traduit en français en 1925, « indique notre désir formel de ne pas perdre de vue les acquisitions passées et de ne pas nous en tenir strictement à ce qu'on appelle de ce non-sens si amusant : le modernisme » (JALOUX, 1931, p. 101). Il s'agit dès lors « de ne pas s'en tenir strictement » au modernisme, catégorie à laquelle Jaloux ne semble pas reconnaître de valeur heuristique. Il convient plutôt, selon lui, d'adopter envers les auteurs Jangada | ano 9, nr. 17, jan/jun, 2021 | ISSN 2317-4722 
étrangers, quelle que soit leur inscription géographique ou temporelle, une attention susceptible de relancer un romantisme européen alors essoufflé et de parvenir ainsi à rassembler les œuvres émanant de trois ensembles distincts : les classiques « historiques », d'abord ; les auteurs contemporains, ensuite, qui publient en Angleterre dans les années 1915-1950 et qui emploient des techniques propres au modernisme ; et, finalement, les auteurs contemporains favorisant une esthétique «classicisante ». C'est exactement ce à quoi procède Jaloux à l'heure de penser et de construire le «roman anglais »: Au pays du roman passe sans ambages de Defoë à Joyce ou de Henry James à Margaret Kennedy ${ }^{5}$. Si l'on saisit bien, à lire l'ouvrage de Jaloux, le caractère inclusif du « roman anglais », genre capable de tenir ensemble des œuvres d'époques et de styles divers, l'on perçoit tout aussi rapidement que le critique subsume cette supposée diversité sous une solide unité, puisqu'il s'efforce d'en faire saillir les constantes, ou pour reprendre ses mots, « des traits essentiels de l'âme anglaise, [que nous] retrouvons avec une si étrange fidélité à travers des périodes si différentes » (JALOUX, 1931, p. 133). L'appariement d'œuvres hétérogènes n'est pas une facilité à laquelle céderait un critique en mal de ligne directrice pour ses compilations d'articles. Tout au contraire, l'assemblage d'entités a priori disparates semble être la norme. Car d'autres concepteurs du genre travaillent à partir de corpus tout à fait comparables à celui de Jaloux. Jacques Vallette, traducteur de Rudyard Kipling et responsable de la chronique anglaise du Mercure de France, procède de la même manière, mêlant lui aussi des romanciers fort divers dans ses réflexions sur le genre « roman anglais $»^{6}$. L'angliciste Irène Simon rend compte, pour sa part, d'un nombre plus restreint d'auteurs, mais dont l'ensemble produit un effet tout aussi disparate ${ }^{7}$.

Le « roman anglais » n'est donc ni tout à fait moderniste ni proprement classique : il agrège des textes que les catégories habituelles séparent. Je l'a déjà dit, la sélection des œuvres se réalise à partir de ce qui se traduit, en français à l'époque, et répond à des préoccupations dictées par le champ francophone. On saisit d'autant mieux pourquoi l'adoption d'un point de

\footnotetext{
${ }^{5}$ Jaloux réunit des textes portant sur Defoë, Thomas Hardy, George Meredith, Henry James, James Joyce, Maurice Baring, Clemence Dane, Katherine Mansfield, Virginia Woolf, Aldous Huxley, Rosamond Lehmann, Margaret Kennedy et W. N. P. Barbellion.

${ }^{6}$ Vallette assemble deux groupes d'auteurs : le premier, qu'il estime être déjà connu du public francophone, réunit Bennett, Butler, Conrad, Forster, Galsworthy, Thomas Hardy, Maugham, Hudson, Kipling, Meredith, Moore, Swinnerton, Wells. Le second est constitué de romanciers contemporains plus confidentiels qu'il convient, selon le critique, de suivre : Baring, Dane, Garnett, Huxley, Joyce, D. H. Lawrence, Rosamund [sic] Lehmann, Mansfield, Hope Mirrlees, Woolf. Voir : "Quelques aspects du roman anglais contemporain », La Quinzaine critique des livres et des revues, vol. 1, nº 10, 25 mars 1930, p. 477-478.

${ }^{7}$ Jane Austen, Emily Brontë, Joseph Conrad, Charles Dickens, George Eliot, Henry Fielding, Thomas Hardy, Henry James, James Joyce, D. H. Lawrence, George Meredith, Samuel Richardson, Walter Scott, Tobias Smollett, Lawrence Sterne, William Thackeray, Virginia Woolf.
} 
vue pragmatique sur le genre s'impose : il permet de comprendre que l'ensemble - le genre « roman anglais »- est modifié par les entrées nouvelles et qu'aucun texte ne peut jamais être considéré comme étant maximalement représentatif du genre, c'est-à-dire qu'aucun texte ne correspond jamais au genre de manière exemplaire et totale. Le « roman anglais », en ce sens, est fait de variations, de modulations et d'accentuations diverses de certains traits stylistiques et culturels. C'est la principale raison pour laquelle « la ressemblance de famille » n'est pas toujours évidente à repérer. Ainsi, pour clarifier et définir quels étaient les traits du « roman anglais » discutés à l'époque, il convient de rechercher, dans les comptes rendus, les ouvrages, les introductions et autres panoramas, les traits que la critique s'accordait à déceler dans les œuvres qui constituaient le genre.

La critique de réception et les spécialistes de littérature anglaise qui ont façonné le « roman anglais » entre les années 1920 et 1950 n'avaient pas en leur possession l'outillage conceptuel (linguistique, narratologique, voire stylistique) qui, on l'a vu à propos de Raimond, ne fut élaboré et diffusé que dans les années 1960. À les lire aujourd'hui, on peut être surpris, comme l'a été Raimond, de leur apparente naïveté, d'une forme d'impressionnisme ou de subjectivisme que trahit parfois un manque de précision dans la description des phénomènes littéraires. Mais si on leur accorde une attention accrue, si on ne préjuge pas de ce qu'on devrait lire, si, en somme, on se départit de ses réflexes critiques pour suivre leurs démonstrations sans les disqualifier d'emblée, d'autres éléments émergent : ils dépassent la simple question technique ou stylistique pour se doubler d'une vigilance aux effets et aux usages du genre. Mis bout à bout, ces éléments confèrent un rôle particulier à la littérature et valorisent un ensemble de croyances relativement différentes de celles que promeut la critique d'aujourd'hui.

Malgré une note d'intention de Jaloux qui écrit, dans Au pays du roman, qu'il faut bien « reconnaître que le roman anglais est actuellement dans un état de magnifique floraison. [Et que s]i l'on voulait d'ailleurs s'y appliquer, on trouverait des traits communs à tous ces livres » (1931, p. 220), il convient de rappeler, encore une fois, que si l'on peut postuler l'idée d'un sens commun sur le genre « roman anglais », c'est-à-dire affirmer qu'il y a bel et bien un accord sur son existence en tant qu'institution ${ }^{8}$, nul ne peut jamais en donner un caractère définitif ou ultime, étant entendu que le genre est toujours en devenir, augmenté régulièrement de nouvelles œuvres qui modifient l'ensemble. Toutefois, au-delà de la particularité de chaque roman et de chaque lecture, la critique de l'époque se retrouve sur certains éléments. De la masse des

\footnotetext{
8 J'entends par là que le genre n'est pas uniquement constitué de normes formelles ou de conventions, mais qu'il résulte d'un accord social sur ce qu'est le genre.
} 


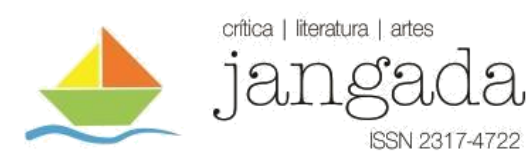

comptes rendus, débats et analyses critiques, émergent en effet trois traits majeurs - le monologue intérieur, l'alternance des points de vue et une attention particulière à la communauté - qui appartiennent non seulement à des types de phénomènes différents, sémantiques, syntaxiques voire thématiques, mais qui ont aussi des conséquences diverses sur la construction du récit et l'implication des lecteurs. Apparaît également, de manière frappante, un appariement opéré par la réception francophone, parfois à la suite des romanciers euxmêmes, entre le « roman anglais » et la philosophie dite « anglo-saxonne », et principalement pragmatiste. Le «moment du roman anglais » coïncide en effet avec une réflexion sur la nature de ce courant philosophique, certes enraciné en Amérique, mais dont certains épigones sont anglais. Si le monde anglophone discute abondamment les thèses de John Dewey, de William James ou de F.C.S. Schiller, leurs travaux sont également traduits en français et reçus par des penseurs comme Émile Durkheim ou Henri Bergson, pour ne citer qu'eux. Il n'est dès lors pas étonnant que la critique de réception du « roman anglais » soit elle aussi marquée par ce courant de pensée qui informe et façonne la définition que les concepteurs du genre du « roman anglais » se font des faits littéraires - certains, comme André Maurois, se donnant même pour mission de rendre compte des travaux de ces philosophes dans des ouvrages hybrides. Résumant le compagnonnage et l'évolution réciproque de la philosophie et du « roman anglais », et ce bien avant que les sartriens ne convoquent systématiquement la philosophie pour éclairer la littérature, le critique Pierre d'Exideuil s'exprime en ces termes : « $[u] n$ des traits marquants de la nouvelle génération [de romanciers anglais] sera son désir de faire progresser de pair littérature et philosophie et de les éclairer l'une par l'autre. » (EXIDEUIL, 1929, p. 568).

Comme l'écrivent Michel Espagne et Michael Werner, « un transfert culturel correspond à une tentative de réinterprétation » (1987, p. 972). C'est peu ou prou à une telle entreprise que s'attèle le monde littéraire francophone lorsqu'il en vient à importer des œuvres anglaises, puis à penser et à décrire le « roman anglais ». Il s'agit pour, les acteurs du champ, de faire sens d'une vague de traduction massive de romans anglais, qu'ils constituent en genre, afin des répondre à des questionnements et à des besoins locaux. La vogue du « roman anglais », permet ainsi au monde littéraire francophone de discuter des fonctions de la littérature et de s'approprier de nouvelles techniques. L'exemple du « roman anglais » suggère que le concept de genre national pourrait contribuer à éclairer les phénomènes de transferts culturels, en restituant, dans les termes qu'utilisaient les acteurs, une partie de ce qu'était la circulation de la littérature dans le long $\mathrm{XX}^{\mathrm{e}}$ siècle, et à ouvrir de nouvelles perspectives aux études sur la littérature mondiale. 


\section{RÉFÉRENCES BIBLIOGRAPHIQUES}

ARMSTRONG, N. Desire and Domestic Fiction : A Political History of the Novel. Oxford : Oxford University Press, 1987.

BELGION, M. Propos sur la littérature anglaise. Paris: Éditions de Flore, 1947.

BOURDIEU, P. Manet : une révolution symbolique. Cours au Collège de France (1998-2000). Paris : Raisons d'agir/ Seuil, 2013.

BOURGET, P. Nouvelles pages de critique et de doctrine. Paris : Plon, 1922.

CAZAMIAN, L. et LEGOUIS, É. Histoire de la littérature anglaise. Paris : Hachette, 1924.

CHARLE, C., « Champ littéraire français et importations étrangères. De la vogue du roman russe à l'émergence d'un nationalisme littéraire (1886-1902) ». In : ESPAGNE, M. et WERNER, M. Philologiques III. Qu'est-ce qu'une littérature nationale? Approches pour une théorie interculturelle du champ littéraire. Paris, Éditions de la Maison des sciences de l'homme, 1994.

CHEVAlLEY, A., Le Roman anglais de notre temps, Oxford : Éditeur de l'Université, 1921.

COHEN, M., The Sentimental Education of the Novel, Princeton: Princeton University Press, 1999.

. «Narratology in the Archive of Literature ». Berkeley. In : Representations, vol. $108, \mathrm{n}^{\circ} 1,2009$, p. 51-75. https://doi.org/10.1525/rep.2009.108.1.51

DELATTRE, F., Le Roman psychologique de Virginia Woolf, Paris : Vrin, 1932.

DIGEON, A. Histoire illustrée de la littérature anglaise. Paris : Didier, 1947.

DOTTIN, P. La Littérature anglaise. Paris : Armand Colin, 1931.

DU BOS, C. Approximations. Paris : Corrêa, 1922-1937.

ELIOT, T.S. «Le roman anglais contemporain ». La Nouvelle Revue française. Paris, $\mathrm{n}^{\circ} 164$, p. 669-675, 1927.

ENGEL, C.-É. Profils anglais. Romanciers de guerre, Charles Morgan, Aldous Huxley, Mazo de La Roche, Rosamund Lehmann, Anne Bridge, Colonel Lawrence, Rex Warner, Stephen Haggard, James Aldridge. Neuchâtel : À la Baconnière, 1946.

Esquisses anglaises. Ch. Morgan, Graham Greene, T. S. Eliot. Paris : Éditions Je sers, 1949. 
ESPAGNE, M. et WERNER, M. « La construction d'une référence culturelle allemande en France : genèse et histoire (1750-1914) ». Annales. Économies, Sociétés, Civilisations. Paris. $\mathrm{n}^{\circ}$ 4, 1987, 969-992. https://doi.org/10.3406/ahess.1987.283428

«L'histoire littéraire et les frontières ». In : DEBAENE, V., MACE, M., JEANNELLE, J.-L. MURAT, M. L'Histoire littéraire des écrivains. Paris : Presses de l’Université Paris-Sorbonne, 2013.

EXIDEUIL, P. d’, « Perspectives anglaises ». Europe. Paris, p. 564-575, 15 avril 1929.

FROW, J. Genre. London ; New York, Routledge: Taylor \& Francis Group, 2015.

GENETTE, G. L'Euvre de l'art. Immanence et transcendance, t.1. Paris : Éditions du Seuil, 1994.

GILLET, L. Esquisses anglaises. Paris : Firmin-Didot et Cie, 1930.

JALOUX, E. Au pays du roman. Corrêa : Paris, 1931.

LALOU, R. Panorama de la littérature anglaise contemporaine. Paris : Éditions Kra, 1926.

LARBAUD, V. Ce vice impuni, la lecture. Domaine anglais. Paris : Gallimard, 1998 [1925 ; 1936].

MACCARTHY, D. «Le roman anglais d'après-guerre (1919-1929) ». Revue de Paris. p. 129$152,1^{\text {er }}$ mai 1932.

MAUROIS, A. Les Anglais. Paris : Éditions des Cahiers libres, 1927. . «Quatre études anglaises ». Paris : Cahiers de la Quinzaine, 1927. . Relativisme. Paris : Éditions Kra, 1930.

1931. . Fragment d'un journal (août-septembre 1930), Relativisme suite. Paris : Sagittaire,

MERCANTON, J. Écrits sur James Joyce. Vevey : L'Aire bleue, 2002.

MORETTI, F. « The spell of indecision ». New Left Review. London, vol. 164, p. 27-33, 1987. . Modern Epic. The World System from Goethe to Garcia Marquez. London ; NewYork : Verso Books, 1996 [1994]. Atlas du roman européen 1800-1900. Paris. Seuil, 2000.

- Graphes, cartes et arbres. Modèles abstraits pour une autre histoire de la littérature. Paris : Les prairies ordinaires, 2008.

Distant Reading, London ; New York: Verso, 2013.

PHILIPPE, G. Le Discours en soi. La représentation du discours intérieur dans les romans de Sartre. Paris : Honoré Champion, 1997. 
RAIMOND, M. La Crise du roman. Des lendemains du Naturalisme aux années vingt. Paris : José Corti, 1966.

ROUSSEAUX, A. Littérature du vingtième siècle. Paris : Albin Michel, 1938.

ROZ, F. Le Roman anglais contemporain. George Meredith, Thomas Hardy, Mme Humphry Ward, Rudyard Kipling, H.G. Wells. Paris: Hachette, 1921.

SAILLENS, É. Esquisse des littératures de langue anglaise. Grande-Bretagne, Irlande, Inde, Dominions, États-Unis. Paris : H. Didier, 1938.

SCHAEFFER, J.-M. Qu'est-ce qu'un genre littéraire? Paris: Seuil, 1989.

SIMON, I. Formes du roman anglais de Dickens à Joyce. Liège : Faculté de philosophie et lettres, 1949.

THÉRENTY, M.-È. et KALIFA, D., «Introduction », Médias 19 [En ligne], Dominique Kalifa et Marie-Ève Thérenty (dir.), Les Mystères urbains au XIXe siècle : Circulations, transferts, appropriations, Publications mis à jour le : 09/03/2015, URL : http://www.medias19.org/index.php?id=21999

THIBAUDET, A. Étranger ou Études de littérature. Genève ; Paris : Éditions de la PetiteFusterie ; Librairie J. Budry \& Cie, 1925.

. «Le roman domestique » [1924]. in Réflexions sur la littérature. Paris, Gallimard, p. 877-892, 2007a.

« Du roman anglais » [1921]. in Réflexions sur la littérature. Paris, Gallimard, p. 589-598, 2007b.

«Le roman urbain » [1924]. in Réflexions sur la littérature. Paris, Gallimard, p. 893-903, 2007c.

VUILLIOMENET, J. «Les femmes et les livres. Cinq romancières anglaises ». Le Mouvement féministe. Genève, n 20, vol. 375, p. 23, 1932.

WILFERT-PORTAL, B. «Cosmopolis et l'homme invisible ». Paris, Actes de la recherche en sciences sociales, vol. 144, $\mathrm{n}^{\circ}$ 1, pp. 33-46, 2002. https://doi.org/10.3917/arss.144.0033

Paris, la France et le reste. . . importations littéraires et nationalisme culturel en France, 1885-1930. 2003, Thèse de doctorat, Université Paris-Sorbonne, Paris. 